\title{
Elevation of $a-1,3$ fucosylation promotes binding ability of TNFR1 to TNF- $a$ and contributes to osteoarthritic cartilage destruction and apoptosis
}

Hanjie Yu

Laboratory for Functional Glycomics, College of Life Sciences, Northwest University Mingxiu Li

The Second Clinical Medical College of Shaanxi University of Chinese Medicine

\section{Xiaodong Wen}

Department of Foot and Ankle Surgery, Honghui Hospital, Xi'an Jiaotong University

Jie Yang

Department of Foot and Ankle Surgery, Honghui Hospital, Xi'an Jiaotong University

\section{Xiaojun Liang}

Department of Foot and Ankle Surgery, Honghui Hospital, Xi'an Jiaotong University

\section{Xia Li}

Laboratory for Functional Glycomics, College of Life Sciences, Northwest University

\section{Xiaojuan Bao}

Laboratory for Functional Glycomics, College of Life Sciences, Northwest University

Jian Shu

Laboratory for Functional Glycomics, College of Life Sciences, Northwest University

Xiameng Ren

Laboratory for Functional Glycomics, College of Life Sciences, Northwest University

Wentian Chen

Laboratory for Functional Glycomics, College of Life Sciences, Northwest University

Zheng Li

Laboratory for Functional Glycomics, College of Life Sciences, Northwest University

Yi Li ( $D$ liyidoctor@163.com )

Department of Foot and Ankle Surgery, Honghui Hospital, Xi'an Jiaotong University

\section{Research Article}

Keywords: osteoarthritis, chondrocyte, a-1,3 fucosylation, FUT10, TNF-a, TNFR1, apoptosis

Posted Date: February 9th, 2022

DOI: https://doi.org/10.21203/rs.3.rs-1328978/v1 
License: (c) (i) This work is licensed under a Creative Commons Attribution 4.0 International License. Read Full License 


\section{Abstract}

Background: Osteoarthritis $(\mathrm{OA})$ is characterized by ECM degradation in chondrocytes and inflammation of the synovial membrane. Fucosylation is a main type of glycosylation, and is involved in a variety of pathological processes, including inflammation and cancer. However, whether fucosylation impacts the OA process is unknown.

Methods: Total proteins were extracted from cartilage samples obtained from patients with $O A(n=11)$, OA rabbit models at different time points $(n=12)$. The OA-associated abnormal glycopatterns were evaluated by lectin microarray and lectin blot. Expression of fucosyltransferases involved in the synthesis of $a-1,3$ fucosylation were assessed by semi-qPCR. The $a-1,3$ fucosylation mediated by FUT10 was interrupted by transfection of siRNA; and the effect of $a-1,3$ fucosylation on OA-associated events was assessed. The TNFR1 antibody microarray was fabricated to investigate the effect of a-1,3 fucosylation on the binding ability of TNFR1 to TNF-a.

Results: Elevated a-1,3 fucosylation is observed in cartilage from OA patients, rabbit models and chondrocytes induced by TNF- $a$ (fold change $>2, p<0.01$ ). The overexpression of FUT10 contributes to this alteration. Downregulation of FUT10 impaired the binding ability of TNFR1 to TNF-a, impeded activation of the NF-KB and P38/JNK-MAPK pathways and eventually retarded ECM degradation, senescence and apoptosis of chondrocytes induced by TNF-a.

Conclusion: The elevation of $a-1,3$ fucosylation is not only a characteristic of OA but also impacts the OA process. Our work provides a new positive feedback loop of "inflammation conditions/TNF-a/FUT10/ a-1,3 fucosylation of TNFR1/NF-KB and P38/JNK-MAPK pathways/proinflammatory processes" that contributes to ECM degradation and apoptosis of chondrocytes.

\section{Background}

Osteoarthritis (OA) is the most common form of degenerative joint disease, affecting millions of people worldwide. The hallmark of osteoarthritic cartilage degeneration is cartilage destruction, subchondral bone remodeling, loss of matrix molecules [1-3]. Some factors such age, gender, obesity, joint injuries and genetic predisposition $[4,5]$. Although $O A$ is considered a disease of purely mechanical cartilage degradation, the etiology of OA is complicated, and molecular alterations of chondrocytes play pivotal role in this process [6]. The proinflammatory cytokines such as interleukin-1b (IL-1 $\beta$ ) and tumor necrosis factor-a (TNF- $\alpha$ ) is increased, and they serve as critical mediators that impair the balance between excessive cartilage damage and cartilage repair process in arthritis [7-9]. Cytokines can activate and increase gene expression of matrix metalloproteinases (MMPs) and aggrecanases (ADAMTS), which digest the components of the cartilage extracellular matrix (ECM) including type II collagen and aggrecan and facilitate cartilage destruction $[10,11]$. Moreover, cytokines also block the synthesis of ECM by suppressing the expression of structural proteins such as type-II collagen and aggrecan [12-14]. 
Glycosylation is one of the most common and complex forms of post-translational modification. In general, glycosylation is critical for maintaining the conformational stability and functionality of proteins [15-17]. Glycoproteins are mainly located at the cell-surface and ECM. Considering that ECM destruction is characteristic of OA, glycosylation may be involved in the development of OA. Several studies have indicated that the aberrant glycosylation of chondrocyte is associated with the OA process. The sialylation and fucosylation of $\mathrm{N}$-glycans were altered in cartilage of $\mathrm{OA}$ animal models, and these alterations preceded histological changes in cartilage [18]. The level of O-GlcNAcylation is increased in $\mathrm{OA}$ cartilage, and the proinflammatory milieu induced by IL-1a can promote the accumulation of $\mathrm{O}$ GlcNAcylated proteins in OA cartilage [19]. Despite the importance described for glycosylation in OA, but the role of the altered glycosylation in the pathophysiology of $O A$ and cartilage degradation is still not fully understood.

Tumor necrosis factor receptor superfamily member $1 \mathrm{~A}$ (TNFR1) is a member of the tumor necrosis factor receptor family. It serves as a receptor of the TNF-a trimer and activates the canonical TNF-a signaling pathway. The death domain of TNFR1 interacts with TNF receptor-associated protein with death domain (TRADD) and recruits Fas-associated protein with death domain (FADD) and caspase-8, consequently leading to activation of caspase-3 and triggering apoptosis [20]. In addition, it also mediates the NF-KB signaling pathway and contributes to inflammation activity in OA cartilage [21]. Glycosylation is vital for the function of TNFR1. The N-glycosylation of TNFR1 could facilitate its ability to bind TNF-a and promote the formation of theTNFa autocrine loop and inflammation in microglia via NF-KB pathways [22]. However, whether the glycosylation of TNFR1 is altered in OA chondrocytes and how the altered glycosylation affects the OA process remain to be illuminated

In the present study, knee articular cartilage from OA patients and animal models was enrolled and the altered glycosylation associated with OA was evaluated by integrated glycomics approaches. Our study demonstrates that thea-1,3 fucosylation which mediated by FUT10 is elevated in OA cartilage and chondrocytes. Interruption of the expression of FUT10 evidently depressed the expression of MMP13 and IL-1 $\beta$ and inhibited chondrocyte apoptosis and senescence induced by TNF- $a$. Interestingly, we demonstrated that the expression of FUT10 was correlated with $\alpha-1,3$ fucosylation of TNFR1 and facilitate its ability to bind to TNF-a, contributing to the activation of the TNFR1 and NF-KB pathways, and eventually promoting the $\mathrm{OA}$ process. Our findings may provide new clues for understanding glycosylation in the pathogenesis of $\mathrm{OA}$, and a new target for therapy.

\section{Materials And Methods}

\section{Collection of Tissue Specimens}

Osteochondral specimens of knee joints were obtained from operations performed from 2020 to 2021 in the Department of Bone Microsurgery, Foot and Ankle Surgery, and Joint Surgery of Xi'an Honghui Hospital. All tissue samples had a final diagnosis provided by clinical specialists. The inclusion criteria

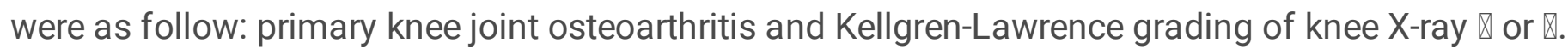


Patients with rheumatoid arthritis, infectious arthritis, traumatic arthritis and other immune system diseases were eliminated from the research samples. As a result, 12 patients were enrolled in the knee osteoarthritis group: all patients were of Han ethnicity Han and they included 5 males and 7 females with an average age of 66.1 years (range: 57-75). All patients underwent knee replacement surgery.

Meanwhile, 11 tibia platform and distal femur comminuted fracture patients were recruited as the control group ( 7 males and 4 females, average age of 41.3 years (range: 24-56)). As the fixed weight-bearing area free bone cartilage block could not be reset in all cases, the pain symptoms of the wounded knee before seeking medical help were compared with the contralateral knee by X-ray and osteoarthritic performance.

\section{Histologic analysis}

To evaluate the degree of degradative changes in the cartilage, cartilaginous tissue from OA patients and rabbit models were embedded in paraffin and sectioned at $6 \mu \mathrm{m}$. After deparaffinization and hydration, the sections were stained with hematoxylin-eosin (HE) and Safranine-O (Solarbio) according to the manufacturer's instructions.

\section{Cell culture and treatment}

The human chondrocyte cell line C28/I2 (C28) was cultured in DMEM/F12 supplemented with 10\% FCS (Gibco Thermo Fisher USA), $100 \mathrm{U} / \mathrm{ml}$ penicillin (Solarbio), and $100 \mu \mathrm{g} / \mathrm{ml}$ streptomycin (Solarbio) in a humidified incubator at $37{ }^{\circ} \mathrm{C}$ in the presence of $5 \% \mathrm{CO}_{2}$. Before treatment, chondrocytes were serumstarved for $12 \mathrm{~h}$ and then stimulated with recombinant human TNF-a (active trimer, acrobiosystems, China) at a concentration of $40 \mathrm{ng} / \mathrm{ml}$ for $48 \mathrm{~h}$, and unstimulated chondrocytes were used as controls synchronously [23]. To investigate the effect of fucosylation on the OA process, 2F-peracetylfucose (2FF, EMD Millipore, Germany) was used to inhibit the biosynthesis of fucosylation in chondrocytes [24]. Briefly, after serum starvation for $12 \mathrm{~h}, 2 \mathrm{FF}$ was added to complete medium at a concentration of $100 \mu \mathrm{M}$ and incubated for $72 \mathrm{~h}$. And then chondrocytes were collected and subjected to further analysis.

\section{Construction of rabbit OA model}

Hulth's modeling method was used to establish the rabbit model of knee osteoarthritis [25]. Briefly, twenty-four male New Zealand white rabbits (purchased from Laboratory Animal Center of Xi'an Jiaotong University, China) were divided into an OA group $(n=12)$ and a sham-operation group $(n=12)$ randomly. At the time of surgery, the rabbits were 3 to 4 months old and had body weights of $2.5 \pm 0.4 \mathrm{~kg}$ and $2.7 \pm 0.3$ $\mathrm{kg}$, respectively (mean $\pm \mathrm{SEM}$ ). Rabbits were anesthetized by $3 \%$ sodium pentobarbital $(1 \mathrm{~mL} / \mathrm{kg}$; Sigma). Intravenous cefazolin (22 mg/kg; Harbin Pharmaceutical Group Pharmaceutical General Factory, China) was administered at the time of the surgical procedure and once every $24 \mathrm{~h}$ for $3 \mathrm{~d}$ postoperatively. A midline skin incision was made over the right knee and a medial parapatellar incision was made through the retinaculum. The medial collateral ligament was sharply divided. A medial parapatellar arthrotomy was performed and the patella dislocated laterally. Care was taken to protect and retract the vascular intraarticular fat pad. With the knee flexed, the anterior cruciate ligament and the posterior cruciate 
ligament were transected. The knee joint was then dislocated to excise the medial meniscus. The joint was irrigated with sterile saline solution. The capsule and the synovium were then closed together with a 4.0 interrupted Vicryl, and the skin was closed. A sham procedure was performed on the right hind limb to serve as a control. For sham surgical controls, right knees were opened as described. After dislocating the patella laterally, the knee was irrigated, but the ligaments and menisci were left intact. Postoperatively, the animals were permitted cage activity without immobilization. The animals were closely monitored for health and welfare. At weeks $0,4,8$ and 12, rabbits from $O A(n=3)$ and sham-operated group $(n=3)$ were euthanized by an intravenous injection of overdose pentobarbital to obtain cartilage samples.

\section{Extraction of Cell/Tissue Protein}

The proteins from chondrocytes and cartilage tissue were extracted by using RIPA Lysis Buffer (Millipore, Billerica, MA) and T-PER Tissue Protein Extraction Reagent (Thermo Fisher Scientific Inc., Rockford, IL, USA) according to the manufacturer's instructions, and $1 \%(\mathrm{~V} / \mathrm{V})$ of protease inhibitor cocktail (Sigma-Aldrich) was added. The protein concentration was determined by BCA assay (Beyotime Biotechnology, Nantong, China).

\section{Lectin Microarray and Data Analysis}

The manufacture of lectin microarray and data acquisition were performed as described previously [2628]. The proteins isolated from cells or tissue were labeled with Cy3 fluorescent dye (GE Healthcare, Biosciences, Piscataway, NJ, USA) and purified using a Sephadex-G25 column (GE Healthcare).

Subsequently, $4 \mu \mathrm{g}$ of labeled protein was applied to the lectin microarrays and incubated in the chamber at $37^{\circ} \mathrm{C}$ for $3 \mathrm{~h}$. After washing and centrifugation, the slides were scanned using a confocal scanner (4000B, AXON Instruments, USA). The fluorescence intensities were extracted by GenePix 7.0 software (Axon). After filtration and normalization, the parallel datasets were compared with each other based upon fold-changes according to the following criteria: fold changes $\geq 1.50$ or $\leq 0.67$ and $p<0.05$ indicated upregulation or downregulation, respectively. Significant difference in lectin between samples were tested by Student's $t$ test.

\section{Lectin blotting}

Lectin blotting was performed as described previously [28-30]. $40 \mu \mathrm{g}$ of protein from OA and control samples was separated by $10 \%$ SDS-PAGE and transferred to PVDF membranes ( $0.22 \mu \mathrm{m}$ Millipore, Bedford, MA, USA). After blocking, the membranes were incubated with Cy 5 labeled lectins overnight at 4 ${ }^{\circ} \mathrm{C}$ and imaged by a STROM Fluorlmager (Molecular Dynamics, Sunnyvale, CA, USA). The gray value of the selected protein bands was measured by ImageJ software $(\mathrm{NIH})$.

\section{Immunoblotting}

Briefly, $10 \mu \mathrm{g}$ of protein was separated by $10 \%$ SDS-PAGE, and then transferred to PVDF membranes (Millipore, Bedford, MA), and blocked with $5 \%$ (w/v) skim milk (Becton Dickinson, Franklin Lakes, NJ) in TBST (TBS buffer with $0.05 \%$ Tween-20, pH 7.6) or 3\% BSA in PBST (for phosphorylated antibodies) for 1 
$\mathrm{h}$ at room temperature. The membranes were probed with primary antibodies overnight at $4{ }^{\circ} \mathrm{C}$ with shaking. The primary antibodies used in this study were as follows: (i) rabbit polyclonal anti-FUT10 (Proteintech, Wuhan, China); (ii) rabbit polyclonal anti-MMP-13 (Proteintech); (iii) rabbit polyclonal antiCOL2A1 (Proteintech); (iv) mouse monoclonal anti-IL-1 $\beta$ (Proteintech); (v) mouse monoclonal anti- NF-KB p65 (Proteintech); (vi) mouse monoclonal anti- phospho-NF-KB p65 (Ser536, CST); (vii) rabbit polyclonal anti-IKBA (Proteintech); (viii) rabbit polyclonal anti-phospho-IKBA (Ser32, CST); (ix) mouse monoclonal anti-P38 MAPK (Proteintech); (x) rabbit monoclonal anti-Phospho-p38 MAPK (Thr180/Tyr182, CST); (xi) mouse monoclonal anti-JNK (Proteintech); (xii) mouse monoclonal phospho-SAPK/JNK (Thr183/Tyr185, CST); (xiii) mouse monoclonal anti-Caspase-8 (Proteintech); (xiv) rabbit polyclonal anti-Caspase-3 (Proteintech); (xv) rabbit polyclonal anti-TNFR1 (Proteintech); (xvi) mouse monoclonal anti- $\beta$ tubulin as internal control (Abways Biotechnology, Shanghai, China). After washing three times with TBST, the membranes were incubated with horseradish peroxidase (HRP)-labeled second antibody (Immunoway, Jiangsu, China) for $2 \mathrm{~h}$ at room temperature with shaking. The membranes were visualized with Immobilon Western chemiluminescent HRP substrate (Millipore, Billerica, MA, USA).

\section{Isolation of RNA and semi-qPCR}

Total RNA from chondrocytes and cartilage tissue was extracted by TRI Reagent (Sigma) according to manufacturer's protocol. Then, $1 \mu \mathrm{g}$ of total RNA was reverse-transcribed using PrimeScript ${ }^{\mathrm{TM}} \mathrm{RT}$ Master Mix (TaKaRa, Japan), and qPCR was performed using a ViiA 7 Real-Time PCR System (Applied Biosystems, USA). SYBR Green based three-step RT-qPCR was performed using TB Green ${ }^{\circledR}$ Premix Ex $\mathrm{Taq}^{\mathrm{TM}}$ II (TaKaRa, Japan). The primer sequences were retrieved from the online PrimerBank database (https://pga.mgh.harvard.edu/primerbank/index.html). The information of primers is summarized in Table S1.

\section{Transfection of small interfering RNA}

Small interfering RNA (siRNA) specific to FUT3, FUT9 and FUT10 were designed with the coding sequences of human by online software DSIR (http://biodev.extra.cea.fr/DSIR/DSIR.html). The information on the siRNAs is shown in Table S2. 2'Ome modified siRNAs and scramble siRNA (negative control) were obtained from GenePharma (Shanghai GenePharma, China). The transfection was performed using HiPerFect reagent (Qiagen, Chatsworth, CA). The cells were harvested $24 \mathrm{~h}$ after transfection, and knockdown effects were evaluated by semi-qPCR. In addition, after transfection for 12 $\mathrm{h}$, the TNF-a (at a final concentration of $40 \mathrm{ng} / \mathrm{mL}$ ) was added to the medium and culture for $48 \mathrm{~h}$. After that, the cells were collected and subjected to follow-up analysis.

\section{Cell proliferation assay}

Cell proliferation was determined by a cell counting kit (CCK-8, YEASEN, Shanghai, China). Briefly, C28 cells were seeded in 96-well plates at concentration of 5,000 cells per well and incubated for $12 \mathrm{~h}$. After treatment, $10 \mu \mathrm{L}$ of CCK8 reagent was added to each well and the cells were incubated at $37^{\circ} \mathrm{C}$ for $1 \mathrm{~h}$. 
The absorbance at $450 \mathrm{~nm}$ of each well was measured by a microplate reader (Bio-Tek Instruments Inc., Winooski, VT, USA). The cell proliferation rates were recorded every $12 \mathrm{~h}$.

\section{SA- $\beta$-gal staining}

Senescence cells were stained using senescence $\beta$-galactosidase staining kit (Beyotime). Briefly, after treatment, the cells were fixed for $15 \mathrm{~min}$ by using stain-fixative. After washing for three times, $1 \mathrm{~mL}$ of staining solution was added to each well, and the plates were incubated at $37^{\circ} \mathrm{C}$ overnight. The SA- $\beta$-gal positive chondrocytes in three random fields of each well were calculated using bright-field microscopy.

\section{Apoptosis assay}

An AnnexinV Alexa Fluor 647/PI apoptosis detection kit (Solarbio) was used to analyze apoptosis. Briefly, the chondrocytes were digested by $0.25 \%$ trypsin (without EDTA, Solarbio). Then, $1 \mathrm{~mL}$ of complete medium was added to neutralize trypsin and the cells were collected and resuspended by cold PBS. After centrifugation, the cells were resuspended in binding buffer, $5 \mu$ of Annexin V/Alexa Fluor 647 was added and incubated at room temperature for $5 \mathrm{~min}$ in the dark $10 \mu \mathrm{L}$ of PI was added and the cells were analyzed on a flow cytometer (ACEA Biosciences, San Diego, USA) using NovoExpress software to detect apoptotic cells.

\section{Immunoprecipitation}

Immunoprecipitation was performed using protein A/G PLUS-Agarose (Santa Cruz, Santa Cruz Biotechnology, Santa Cruz, CA, USA) according to manufacturer's protocol with modifications. In order to protect the activity of TNFR1, the low pH elution buffer $(100 \mathrm{mM}$ glycine, $50 \mathrm{mM}$ Tris- $\mathrm{HCl}$, and $500 \mathrm{mM}$ $\mathrm{NaCl}, \mathrm{pH} 2.0)$ was used to elute TNFR1, and $10 \mu \mathrm{L}$ of Tris buffer $(1 \mathrm{M}, \mathrm{pH} 9.5)$ was added to neutralize the low $\mathrm{pH}$ condition.

\section{Manufacture of antibody microarray}

To investigate the effect of fucosylation on the binding capability of TNFR1 to TNF-a, a TNFR1 antibody microarray was fabricated. The antibodies were diluted with printing buffer (PBST with $0.01 \%(w / v) B S A$ ) to a concentration of $200 \mathrm{ng} / \mu \mathrm{L}$ and spotted using an arrayer (SmartArrayer 48, Capitalbio). After blocking, the enrichment of TNFR1 from different sources was diluted to $0,5,10$ and $20 \mathrm{ng} / \mu \mathrm{L}$ and applied to microarrays and incubated at $25^{\circ} \mathrm{C}$ overnight. Then, the slides were incubated with TNF-a (20 $\mathrm{ng} / \mu \mathrm{L}$ ) and $10 \mathrm{ng} / \mu \mathrm{L}$ of primary antibody against TNF-a (Cy3 labeled, Bioss) for $3 \mathrm{~h}$ respectively. The net fluorescence intensities (the raw fluorescence intensities - background) of each sopt was acquired by Genepix 7.0 software (Axon Instruments, Inc. USA). To compare the difference in binding signals, we set the binding signals at $0 \mathrm{ng} / \mu \mathrm{L}$ TNFR1 as the baseline, and the differences in binding signals of TNFR1 Ab between TNF-a treated chondrocytes, siRNA-FUT10 transfected chondrocytes and controls were tested by one-way ANOVA. 


\section{Results}

\section{Assessment of abnormal glycopatterns in cartilage from OA patients}

To investigate the abnormal glycosylation associated with $O A$, the glycan profile of cartilage from $O A$ patients and normal controls was evaluated by lectin microarray. As shown in Fig. 1a and 1b, the joint morphology and space were altered in OA patients. Moreover, abundant osteophytes osteophyte, sclerosis of subchondral bone, cystic changes in the proximal medial tibia and varus deformities were observed in the knee-joints of OA patients. The staining results revealed that the OA tissue slices displayed common OA changes, including discontinuity of the cartilage surface and non-uniform distribution of chondrocytes (Fig. 1c-e). The binding of several lectins such as PSA and PHA-E showed distinct differences between $\mathrm{OA}$ and normal cartilage, suggesting that glycosylation of cartilage was altered in OA patients (Fig. 1g, h). The average normalized fluorescent intensities (NFIs) of all lectins from $\mathrm{OA}$ and control samples were compared (summarized as the mean values \pm SD in Table S3). As a result, the NFIs of 15 lectins (e.g., PSA, AAL and LTL) were significantly altered between OA and normal cartilage (Fig. 1i). Notably, the $\alpha-1,3 / 6$ fucosylation level which was identified by PSA, AAL and LTL was significantly increased in OA cartilage compared with normal controls (fold change $>2, p<0.01$ ).

As a results of lectin blotting, PSA and LTL showed distinct binding to two apparent bands (approximately $100 \mathrm{kDa}$ and $60 \mathrm{kDa}$ ) and AAL showed distinct binding to two apparent bands (approximately $60 \mathrm{kDa}$ and $70 \mathrm{kDa}$ ) in $\mathrm{OA}$ cartilage (Fig. $1 \mathrm{j}, \mathrm{k}$ ). These results demonstrated that glycosylation, especially a-1,3/6 fucosylation was significantly altered in the cartilage of OA patients.

\section{The level of fucosylation increased in cartilage of the OA model}

During the development of $\mathrm{OA}$, the medial joint space narrowed, the cartilage was slightly injured, and the cartilage surface was not smooth but rather contoured at 4 weeks. The cartilage was moderately damaged, the distribution of chondrocytes was disordered, macro-cracks on the surface of cartilage and high-density shadows were observed in joints at 8 weeks. The joint space was significantly narrowed, osteophyte and joint deformities and advanced OA lesions, including thin hardened and rough cartilage layers, disappearance of the abnormal tide line of subchondral bone and severe loss of Safranin-O staining and clefts were observed in OA rabbits at 12 weeks postoperatively (Fig. 2a).

As result of lectin microarrays, 34 lectins showed significantly altered NFIs during the OA process (Table S4). Combined with the results of OA patients (Table 1), PSA showed significantly increased NFIs in all OA models compared with the control. WGA and AAL showed significantly increased NFIs, and the high mannose-type N-glycan binder ConA showed significantly decreased NFIs in OA models 4 weeks postoperation compared with the sham-operated controls. However, the relative abundance of Fuca1-3Galß1-4GICNAc which is recognized by LTL, did not show a distinct difference between OA models and controls at the early stage of OA but exhibited a significant increase in the middle and late stages of OA models compared with controls (Fig. 2b). Our findings indicated that an increased level of a1-3/ 6 fucosylation is associated with the OA process. 


\section{Suppressed synthesis of fucosylation retarded ECM destruction}

Next, we primarily investigated whether fucosylation impacts OA-associated events. After treatment with TNF-a, fucosylation was evidently increased, as indicated by the NFIs of AAL, LTL and PSA (Fig. 3a). Subsequently, we used 2FF to interrupt the biosynthesis of fucosylation in chondrocytes with or without TNF-a treatment. Consequently, the expression of COL2A1 was decreased and MMP13 was increased after treatment with TNF-a, which is a common event in OA. However, after treatment with 2FF, the expression of COL2A1 was distinctly increased and MMP-13 was decreased compared with TNF-astimulated cells (Fig. 3b). Furthermore, the staining indicated that the number of SA- $\beta$-gal positive cells was significantly increased in chondrocytes treated with TNF- $a$; however, the number of senescent cells was significantly decreased after treatment with 2FF compared with TNF-a-stimulated cells. T (Fig. 3c, d). However, the result of cell viability assay indicated that 2FF did not impact chondrocyte proliferation (Fig. $3 e)$. These findings indicated that suppression of fucosylation inhibits ECM degradation and cell senescence caused by TNF-a.

\section{Loss of FUT10 impeded ECM degradation and apoptosis induced by TNF-a}

The biosynthesis of fucosylation is catalyzed by fucosyltransferases (FUTs). Hence, we primarily investigated the alterations in FUTs in chondrocytes stimulated with TNF-a. There was a significant increase in the levels of FUT3, FUT9 and FUT10 in chondrocytes treated with TNF-a compared with control (Fig. 4a). In parallel, we reanalyzed fucosylation related gene expression patterns in human OA and non-OA knee lateral and medial tibial plateaus from the Gene Expression Omnibus database (GEO GSE51588) [31]. As results of GEO data and our findings, it demonstrated that the transcription and expression of FUT10 were significantly increased in OA cartilage compared with controls (Fig. 4 b-d).

Next, we investigated whether the synthesis of $a-1,3$ fucosylation mediated by FUT10 could impact the molecular events associated with OA. After transfection, the expression of FUT10 was significantly reduced (Fig. 4e). However, blocking FUT10 gene expression had no obvious effect on cell proliferation (Fig. 4f). The number of SA- $\beta$-Gal positive cells and the proportion of apoptotic cells were significantly reduced in chondrocytes pretransfected with FUT10 siRNA compared with TNF-a-treated cells. (Fig. 4g-4i). In cultured chondrocytes, TNF-a remarkably increased the expression of molecules associated with cartilage degeneration, inflammation and apoptosis including MMP-13, IL-1 $\beta$, caspase-3 and caspase-8. However, the expression of these OA- associated proteins was suppressed in TNF-ainduced chondrocytes pretreated with FUT10 siRNA. Moreover, pretransfection of FUT10 siRNA attenuated TNF-a-mediated plkB-a, p-p65, p-p38 and p-JNK expression suggesting that downregulation of FUT10 expression suppresses the activation of the NF-KB and p38-JNK pathways in OA chondrocytes (Fig. 4j).

\section{The fucosylation of TNFR1 affected the binding of TNF- $a$}

TNFR1 is not only the membrane receptor for TNF-a but also recruits the adaptors FADD and caspase-8 upon binding of the ligand TNF-a to initiate apoptosis [32]. Hence, we explored whether the expression or 
glycosylation of TNFR1 are altered in TNF-a stimulated cells. We found that the expression of TNFR1 was slightly elevated in TNF-a-treated cells compared with control cells but did not find significant difference in chondrocytes pretransfected with FUT10 siRNA (Fig. 5a). Subsequently, we performed immunoprecipitation and lectin blotting to investigate the fucosylation of TNFR1 in TNF-a-stimulated chondrocytes. After immunoprecipitation, the protein level of TNFR1 did not show a difference, but a-1,3 fucosylation was increased in TNF-a-treated chondrocytes and cartilage from OA patients compared with controls (Fig. 5b, c). However, a-1,3 fucosylation was decreased in TNF-a-treated chondrocytes, indicating downregulation of FUT10 could inhibit the $\mathrm{a}-1,3$ fucosylation of TNFR1 without impacting protein expression (Fig. 5b).

Subsequently, a TNFR1 antibody microarray was used to investigate the role of $a-1,3$ fucosylation of TNFR1 in the binding ability of TNFR1 to TNF-a (Fig. 5d). The results indicated that the elevated a-1,3 fucosylation of TNFR1 significantly enhanced the binding ability to TNF-a, and downregulation of FUT10 significantly impaired the binding ability (Fig. $5 e, f)$. These findings indicated that the $a-1,3$ fucosylation of TNFR1 mediated by FUT10 could impact the binding ability of TNFR1 to TNF-a.

\section{Discussion}

$\mathrm{OA}$ is the most common types of arthritis worldwide and is characterized by ECM degradation through the upregulation of MMPs and elevated levels of proinflammatory cytokines such as TNF-a, IL-1 $\beta$ and COX-2 [33,34]. One of the most fascinating aspects of glycosylation is heterogeneity. The structural heterogeneity of glycans has been observed in various diseases, and it also contributed to the process. In the present study, we observed that several types of glycosylation were altered in OA patients and animal models, including elevated levels of a-1, 3/6 fucosylation, galactosylation and decreased levels of high mannose-type N-glycans and bisecting GICNAc. Several studies have reported that the glycosylation of IgG and synovial fluid is altered during the OA process $[35,36]$. In addition, it reported that the alteration of glycosylation in a variety of proteins of chondrocytes playing an important role in degenerative changes of chondrocytes and contributing to the initiation and progression of $\mathrm{OA}[37,38]$.

Altered fucosylation has been observed in a number of inflammatory conditions, and inhibition of fucosylation could modulate human nucleus pulposus cell protein translation of catabolic enzymes in response to inflammation $[39,40]$. Our findings revealed that $a-1,3 / 6$ fucosylation is characteristic of OA chondrocytes. Importantly, inhibiting the biosynthesis of fucosylation by $2 \mathrm{FF}$ could retard ECM degradation and senescence induced by TNF-a. The Lewis antigen is a fucosylated carbohydrate moiety, located at terminal of N/O-glycans and glycosphingolipids. FUT10 is a member of the a-1, 3 fucosyltransferase family which responds to the synthesis of Lewis $X$. The sialylated Lewis $X$ is known as an inflammation-associated antigen. We found that FUT10 is overexpressed in OA cartilage and chondrocytes induced by TNF-a; moreover, downregulation of FUT10 could reduce the expression of inflammatory cytokine and ECM degradation. Chronic low-grade inflammation plays an important role in the development of OA. The expression of Lewis $\mathrm{X}$ antigen in synovial tissue is correlated with histological OARSI grades of OA, which indicates an association of increased inflammatory activity with 
advanced cartilage degeneration [41]. Sialyl Lewis X promotes synovial lubricin binding to polymorphonuclear granulocytes in an L-selectin-dependent and L-selectin-independent manner and may play a role in polymorphonuclear granulocytes-mediated inflammation in rheumatic arthritis [42].

Proinflammatory cytokines play vital roles in catabolic reactions of arthritic cartilage [43]. As a key inflammatory cytokine, TNF- $a$ is produced by activated synoviocytes, mononuclear cells or articular cartilage itself. TNF-a promotes the expression of matrix metalloproteinases such as MMPs ADAMTS-4, 5 and 7, as well as other inflammatory cytokines, and contributes to cartilage degeneration in OA development $[44,46]$. TNFR1 is cell surface receptor of TNF- $a$. The binding of TNF to TNFR1 triggers a series of intracellular events that ultimately result in the activation of the NF-KB and p38/JNK MAPK signal pathways [46]. The N-glycans are pivotal for the TNF-TNFR1 interaction and subsequent NF-KB activation [47]. The a-2,6 sialylation of TNFR1 mediated by ST6Gal-I was found to inhibit TNF-induced TNFR1 internalization and apoptosis [48]. Our results demonstrated that depressed expression of FUT10 not only reduced the a-1,3 fucosylation level of TNFR1, but also impaired the binding capacity to TNF-a and restrained the activation of the NF-KB and p38/JNK MAPK signaling pathways downstream of TNFR1. The activation of the canonical NF-KB pathway is mediated by proinflammatory cytokine (such as TNF- $\mathrm{a}$ ). As NF-KB heterodimers translocate into the nucleus, the expression of a variety of proteins is triggered, such as metalloproteinases, NF-KB-mediated catabolic cytokines and chemokines, which promote inflammation and apoptosis of OA chondrocytes [49]. The p38/JNK-MAPK pathway was reported to be correlated with chronic inflammation. Once activated, downstream transcription factors upregulate the expression of genes relevant to $\mathrm{OA}$ including proinflammatory cytokines and matrixdegrading enzymes such as MMPs [50]. Moreover, the prediction results revealed that Elk-1 and MEF-2 are potential transcription factors for FUT10, which are downstream transcription factors of the P38/JNKMAPK pathway. Collectively, our results suggested that the inflammatory condition of OA chondrocytes facilitates the expression of TNF- $a$, which leads to the upregulation of FUT10. Then, the $a-1,3$ fucosylation of TNFR1 mediated by FUT10 is elevated and promotes the binding ability to TNF-a. Consequently, the NF-KB and P38/JNK-MAPK pathways are activated and promote inflammation and apoptosis of OA chondrocytes. In addition, the transcription factors Elk-1 and MEF-2 which are downstream of the P38/JNK-MAPK pathway may translocate into nucleus and up-regulate of FUT10.

\section{Conclusions}

Overall, our study revealed that the FUT10-mediated increase a-1,3 fucosylation is characteristic of OA cartilage and chondrocytes. Moreover, the $\mathrm{a}-1,3$ fucosylation level of TNFR1 mediated by FUT10 impacted the binding ability to TNF-a. Suppressed expression of FUT10 impedes activation of the NF-KB and P38/JNK-MAPK pathways and retards ECM degradation, senescence and apoptosis of chondrocytes induced by TNF-a. Our work provides a new positive feedback loop of inflammation conditions/TNFa/FUT10/a-1,3 fucosylation of TNFR1/NF-KB and P38/JNK-MAPK pathways/proinflammatory processes that contribute to ECM degradation and apoptosis of OA chondrocytes and eventually accelerates $O A$ process. 


\section{Abbreviations}

OA: Osteoarthritis, IL-1 $\beta$ : interleukin-1b, MMPs: matrix metalloproteinases, ADAMTS: aggrecanases, ECM: extracellular matrix, TNF-a: Tumor necrosis factor-a, TNFR1: Tumor necrosis factor receptor superfamily member 1A, TRADD: TNF receptor-associated protein with death domain, FADD: Fas-associated protein with death domain, HE: hematoxylin-eosin, 2FF: 2F-peracetyl-fucose, siRNA: Small interfering RNA, FUTs: fucosyltransferases, GEO: Gene Expression Omnibus database.

\section{Declarations}

\section{Ethics approval and consent to participate}

The collection of human tissues was performed in accordance with approved guidelines and approved by the human ethics committee of all participating units (Xi'an Honghui Hospital, Shannxi). Written informed consent was received from the participants. The protocols of construction of OA rabbit model were approved by the Institutional Animal Care and Use Committee of Xi'an Honghui Hospital, China. This study was conducted in accordance with the ethical guidelines of the Declaration of Helsinki.

\section{Consent for publication}

Written informed consent was obtained from all participants.

\section{Availability of data and materials}

The data used to support the fndings of this study are available from the corresponding author upon request.

\section{Competing interests}

None of the authors have any competing interest to declare.

\section{Funding}

This study was supported by Shaanxi science and technology research and development plan project (Grant No. 2016sf-333); Xi'an health bureau scientific research project (Grant No. j201802027); China Postdoctoral Science Foundation (Grant No. 2020M673628XB) and the National Natural Science Foundation of China (Grant No. 32101030).

\section{Author contributions}

All authors were involved in drafting the article or data analysis, and all authors approved the final version to be published. HJY, MXL, ZL and YL: designed research, analyzed data and wrote the paper. XDW, JY and XJL: collected cartilage samples. XL and XJB: performed majority of the experiments. JS, XMR and WTC: analyzed data. HJY and MXL contributed equally to this study. 


\section{Acknowledgments}

We thank Dr. Hao Yang (the director of experimental center of Honghui Hospital) for technical assistance and Tiezeng Zhang (Center for Endemic Disease of Xi'an Jiaotong University) for the assistance of histopathology. We also thank AJE for language polishing.

\section{References}

1. Loeser RF, Goldring SR, Scanzello CR, Goldring MB. Osteoarthritis: a disease of the joint as an organ. Arthritis Rheum. 2012;64(6):1697-1707.

2. Yang CY, Chanalaris A, Troeberg L. ADAMTS and ADAM metalloproteinases in osteoarthritis - looking beyond the 'usual suspects'. Osteoarthritis Cartilage. 2017;25(7):1000-9.

3. Fang H, Huang L, Welch I, Norley C, Holdsworth DW, Beier F, et al. Early Changes of Articular Cartilage and Subchondral Bone in The DMM Mouse Model of Osteoarthritis. Sci Rep. 2018;8(1):2855.

4. Palazzo C, Nguyen C, Lefevre-Colau MM, Rannou F, Poiraudeau S. Risk factors and burden of osteoarthritis. Ann Phys Rehabil Med. 2016;59(3):134-8.

5. Kulkarni K, Karssiens T, Kumar V, Pandit H. Obesity and osteoarthritis. Maturitas. 2016;89:22-8.

6. Glyn-Jones S, Palmer AJ, Agricola R, et al. Osteoarthritis. Lancet. 2015;386(9991):376-87.

7. Jenei-LanzI Z, Meurer A, Zaucke F. Interleukin-1 $\beta$ signaling in osteoarthritis - chondrocytes in focus. Cell Signal. 2019;53:212-23.

8. Wang $X$, Hunter DJ, Jin X, Ding C. The importance of synovial inflammation in osteoarthritis: current evidence from imaging assessments and clinical trials. Osteoarthritis Cartilage. 2018;26(2):165-74.

9. Kapoor M, Martel-Pelletier J, Lajeunesse D, Pelletier JP, Fahmi H. Role of proinflammatory cytokines in the pathophysiology of osteoarthritis. Nat Rev Rheumatol. 2011;7(1):33-42.

10. Wilkinson DJ, Desilets A, Lin H, Charlton S, Del Carmen Arques M, et al. The serine proteinase hepsin is an activator of pro-matrix metalloproteinases: molecular mechanisms and implications for extracellular matrix turnover. Sci Rep. 2017;7(1):16693.

11. Xue J, Wang J, Liu Q, Luo A. Tumor necrosis factor-a induces ADAMTS-4 expression in human osteoarthritis chondrocytes. Mol Med Rep. 2013;8(6):1755-60.

12. Molnar V, Matišić V, Kodvanj I, Bjelica R, Jeleč Ž, et al. Cytokines and Chemokines Involved in Osteoarthritis Pathogenesis. Int J Mol Sci. 2021;22(17):9208.

13. Chen C, Xie J, Rajappa R, Deng L, Fredberg J, Yang L. Interleukin-1 $\beta$ and tumor necrosis factor-a increase stiffness and impair contractile function of articular chondrocytes. Acta Biochim Biophys Sin (Shanghai). 2015;47(2):121-9.

14. Livshits $\mathrm{G}$, Kalinkovich A. Hierarchical, imbalanced pro-inflammatory cytokine networks govern the pathogenesis of chronic arthropathies. Osteoarthritis Cartilage. 2018;26(1):7-17.

15. Xiong Y, Karuppanan K, Bernardi A, Li Q, Kommineni V, et al. Effects of N-Glycosylation on the Structure, Function, and Stability of a Plant-Made Fc-Fusion Anthrax Decoy Protein. Front Plant Sci. 
2019;10:768.

16. Xu C, Ng DT. Glycosylation-directed quality control of protein folding. Nat Rev Mol Cell Biol. 2015;16(12):742-52.

17. Jayaprakash NG, Surolia A. Role of glycosylation in nucleating protein folding and stability. Biochem J. 2017;474(14):2333-47.

18. Matsuhashi T, Iwasaki N, Nakagawa $\mathrm{H}$, et al. Alteration of $\mathrm{N}$-glycans related to articular cartilage deterioration after anterior cruciate ligament transection in rabbits. Osteoarthritis Cartilage. 2008;16(7):772-8.

19. Tardio L, Andrés-Bergós J, Zachara NE, et al. O-linked N-acetylglucosamine (O-GIcNAc) protein modification is increased in the cartilage of patients with knee osteoarthritis. Osteoarthritis Cartilage. 2014;22(2):259-63.

20. Micheau O, Tschopp J. Induction of TNF receptor I-mediated apoptosis via two sequential signaling complexes. Cell. 2003;114(2):181-90.

21. Qu R, Chen X, Wang W, et al. Ghrelin protects against osteoarthritis through interplay with Akt and NFkappaB signaling pathways. FASEB J. 2018;32(2):1044-58.

22. Han L, Zhang D, Tao T, Sun X, Liu X, Zhu G, et al. The role of N-glycan modification of TNFR1 in inflammatory microglia activation. Glycoconj J. 2015;32(9):685-93.

23. Zhang X, Prasadam I, Fang W, Crawford R, Xiao Y. Chondromodulin-1 ameliorates osteoarthritis progression by inhibiting HIF-2a activity. Osteoarthritis Cartilage. 2016;24(11):1970-80.

24. Li J, Guillebon AD, Hsu JW, Barthel SR, Dimitroff CJ, Lee YF, et al. Human fucosyltransferase 6 enables prostate cancer metastasis to bone. Br J Cancer. 2013;109(12):3014-22.

25. Hulth A, Lindberg L, Telhag H. Experimental osteoarthritis in rabbits. Preliminary report. Acta Orthop Scand. 1970;41(5):522-30.

26. Yu H, Zhu M, Qin Y, Zhong Y, Yan H, Wang Q, et al. Analysis of glycan-related genes expression and glycan profiles in mice with liver fibrosis. J Proteome Res. 2012;11(11):5277-85.

27. Shu J, Yu H, Li X, Zhang D, Liu X, Du H, et al. Salivary glycopatterns as potential biomarkers for diagnosis of gastric cancer. Oncotarget. 2017;8(22):35718-27.

28. Liu X, Yu H, Qiao Y, Yang J, Shu J, Zhang J, et al. Salivary Glycopatterns as Potential Biomarkers for Screening of Early-Stage Breast Cancer. EBioMedicine. 2018;28:70-9.

29. Qin Y, Zhong Y, Zhu M, Dang L, Yu H, Chen Z, Chen W, et al. Age- and sex-associated differences in the glycopatterns of human salivary glycoproteins and their roles against influenza A virus. J Proteome Res. 2013;12(6):2742-54.

30. Zhong Y, Qin Y, Yu H, Yu J, Wu H, Chen L, et al. Avian influenza virus infection risk in humans with chronic diseases. Sci Rep. 2015; 5:8971.

31. Chou CH, Wu CC, Song IW, Chuang HP, Lu LS, Chang JH, et al. Genome-wide expression profiles of subchondral bone in osteoarthritis. Arthritis Res Ther. 2013;15(6):R190.

32. Barnhart BC, Peter ME. The TNF receptor 1: a split personality complex. Cell. 2003;114(2):148-50. 
33. Kapoor M, Martel-Pelletier J, Lajeunesse D, Pelletier JP, Fahmi H. Role of proinflammatory cytokines in the pathophysiology of osteoarthritis. Nat Rev Rheumatol. 2011;7(1):33-42.

34. Wang P, Guan PP, Guo C, Zhu F, Konstantopoulos K, Wang ZY. Fluid shear stress-induced osteoarthritis: roles of cyclooxygenase-2 and its metabolic products in inducing the expression of proinflammatory cytokines and matrix metalloproteinases. FASEB J. 2013;27(12):4664-77.

35. Sun D, Hu F, Gao H, Song Z, Xie W, Wang P, Shi L, et al. Distribution of abnormal IgG glycosylation patterns from rheumatoid arthritis and osteoarthritis patients by MALDI-TOF-MSn. Analyst. 2019;144(6):2042-51.

36. Albrecht S, Unwin L, Muniyappa M, Rudd PM. Glycosylation as a marker for inflammatory arthritis. Cancer Biomark. 2014;14(1):17-28.

37. Tardio L, Andrés-Bergós J, Zachara NE, Larrañaga-Vera A, Rodriguez-Villar C, Herrero-Beaumont G, et al. O-linked N-acetylglucosamine (O-GIcNAc) protein modification is increased in the cartilage of patients with knee osteoarthritis. Osteoarthritis Cartilage. 2014;22(2):259-63.

38. Urita A, Matsuhashi T, Onodera T, Nakagawa H, Hato M, Amano M, et al. Alterations of high-mannose type N-glycosylation in human and mouse osteoarthritis cartilage. Arthritis Rheum. 2011;63(11):3428-38.

39. Li J, Hsu HC, Mountz JD, Allen JG. Unmasking Fucosylation: from Cell Adhesion to Immune System Regulation and Diseases. Cell Chem Biol. 2018;25(5):499-512.

40. Joyce K, Mohd Isa IL, Krouwels A, Creemers L, Devitt A, Pandit A. The role of altered glycosylation in human nucleus pulposus cells in inflammation and degeneration. Eur Cell Mater. 2021;41:401-20.

41. Koller U, Waldstein W, Krenn V, Windhager R, Boettner F. Varus knee osteoarthritis: Elevated synovial CD15 counts correlate with inferior biomechanical properties of lateral-compartment cartilage. J Orthop Res. 2018;36(3):841-6.

42. Jin C, Ekwall AK, Bylund J, Björkman L, Estrella RP, Whitelock JM, et al. Human synovial lubricin expresses sialyl Lewis $\mathrm{x}$ determinant and has L-selectin ligand activity. J Biol Chem. 2012;287(43):35922-33.

43. Guo B, Yang N, Borysiewicz E, Dudek M, Williams JL, Li J, et al. Catabolic cytokines disrupt the circadian clock and the expression of clock-controlled genes in cartilage via an NFKB-dependent pathway. Osteoarthritis Cartilage. 2015;23(11):1981-8.

44. Qu R, Chen X, Wang W, Qiu C, Ban M, Guo L, et al. Ghrelin protects against osteoarthritis through interplay with Akt and NF-kB signaling pathways. FASEB J. 2018;32(2):1044-58.

45. Miller RE, Miller RJ, Malfait AM. Osteoarthritis joint pain: the cytokine connection. Cytokine. 2014;70(2):185-93.

46. Hosseinzadeh A, Kamrava SK, Joghataei MT, Darabi R, Shakeri-Zadeh A, Shahriari M, et al. Apoptosis signaling pathways in osteoarthritis and possible protective role of melatonin. J Pineal Res. 2016;61(4):411-25.

47. Moriwaki K, Chan FKM, Miyoshi E. Sweet modification and regulation of death receptor signalling pathway. J Biochem. 2021;169(6):643-52. 
48. Holdbrooks AT, Britain CM, Bellis SL. ST6Gal-I sialyltransferase promotes tumor necrosis factor (TNF)-mediated cancer cell survival via sialylation of the TNF receptor 1 (TNFR1) death receptor. $J$ Biol Chem. 2018;293(5):1610-22.

49. Rigoglou S, Papavassiliou AG. The NF-kB signalling pathway in osteoarthritis. Int J Biochem Cell Biol. 2013;45(11):2580-4.

50. Ge HX, Zou FM, Li Y, Liu AM, Tu M. JNK pathway in osteoarthritis: pathological and therapeutic aspects. J Recept Signal Transduct Res. 2017;37(5):431-36.

\section{Tables}

Table 1. The lectins showed significant altered NFIs in OA models and patients compared to controls

Lectin Specificity
OA models /

sham-operated groups

\begin{tabular}{|c|c|c|c|c|c|c|}
\hline & & & & & & \\
\hline & & $\begin{array}{l}0 \\
\text { weeks/ } \\
\text { control }\end{array}$ & $\begin{array}{l}4 \\
\text { weeks/ } \\
\text { controls }\end{array}$ & $\begin{array}{l}8 \\
\text { weeks/ } \\
\text { controls }\end{array}$ & $\begin{array}{l}12 \\
\text { weeks/ } \\
\text { controls }\end{array}$ & \\
\hline PSA & $\begin{array}{l}\text { Fuca-1,6GlcNAc, a-D-Man, a- } \\
\text { D-Glc }\end{array}$ & $2.586^{\star}$ & $3.272^{\star \star}$ & $2.489 * \star$ & $5.104^{\star \star \star}$ & $3.585^{\star \star \star}$ \\
\hline AAL & a-Fucose & / & $2.261^{\star}$ & $2.064^{*}$ & $1.976^{\star}$ & $2.322^{\star \star}$ \\
\hline WGA & $(\mathrm{GlcNAc})_{\mathrm{n}}$ and multivalent Sia & / & $1.791^{*}$ & $2.693^{*}$ & $2.155^{\star \star}$ & $2.309 * * *$ \\
\hline LTL & $\begin{array}{l}\text { Fuca1-3Galß1-4GIcNAc, } \\
\text { Fuca1-anti-H blood group } \\
\text { specificity }\end{array}$ & / & / & $2.339 * *$ & $3.084^{\star \star}$ & $2.196 * \star$ \\
\hline ConA & High-Mannose type N-glycans & / & $0.484^{*}$ & $0.408^{*}$ & $0.556^{*}$ & $0.410 * \star \star$ \\
\hline
\end{tabular}

OA patients / normal controls

/ no significant difference, ${ }^{*} p<0.05, * \star p<0.01$, and ${ }^{\star \star *} p<0.001$

\section{Figures}

\section{Figure 1}

Evaluation of altered glycopatterns in cartilage from OA patients. (a-b) The morphology of knee joint articular was assessed by X-ray image. Compared to normal control (a), the joint space and morphology of OA patient (b) were altered. (c-d) The articular cartilage from normal control (c) and OA patients (d) were evaluated for histopathologic features using HE staining. (e-f) Safranin-O staining was used to 
assessed the degenerative degree of cartilage section in normal control (e) and OA patients (f) (original magnification $\times 100)$. $(\mathbf{g}-\mathbf{h})$ Scanned images are obtained for the analysis of glycopatterns of articular cartilage from OA patients (g) and normal controls (h). (i) The lectins with increased NFIs in OA patients are marked with red boxes, and those with decreased NFIs are marked with white boxes. The NFIs of 10 lectins were significantly changed in OA patients $(n=12)$ compared to normal controls $(n=11)$ based on fold change and $t$-test, the data were presented as the averaged NFI $\pm \operatorname{SD}\left(* p<0.05\right.$, ** $p<0.01$, and ${ }^{\star * \star} p$ $<0.001)$. (j) Lectin blotting of PSA, LTL and AAL were performed to validate of the differential expressions of the glycopatterns in cartilage from OA patients and normal controls, the difference protein bands between OA patients and normal controls were marked with red frames. $(\mathbf{k})$ The gray value of the difference protein bands was measured using the ImageJ software.

\section{Figure 2}

The fucosylation level was raised accompanied by development of OA. The OA ribbit model was established to assess the dynamic change of glycopatterns in cartilage during the development of OA. (a) The X-ray image was used to evaluate the morphology of knee joints from OA models at $0,4,8$, and 12 weeks after surgery (upper). The cartilage tissue sections were obtained from the right knee joints of $O A$ models and stained with HE (middle) and Safranin-O (lower) for the indicated time periods (original magnification $\times 100$ ). (b) The NFIs of 5 lectins were significantly altered in OA models ( $n=3$ per time point) compared to sham operation controls ( $\mathrm{n}=3$ per time point) based on fold change and $t$-test. (NS: not significantly different, NS: no significant difference, ${ }^{*} p<0.05$, ${ }^{\star *} p<0.01$, and ${ }^{* \star *} p<0.001$ ).

\section{Figure 3}

Suppression of the synthesis of fucosylation impeded ECM degradation and cell senescence. (a) The a-1,3/ 6 fucosylation level which identified by AAL, LTL and PSA was markedly elevated in chondrocytes induced by TNF-a compared to control. (b) Effect of fucosylation synthesis inhibitor 2FF alone or in combination with TNF-a on inducible expression of COL2A1 and MMP-13. $\beta$-tubulin is internal control. (c) SA- $\beta-G$ al staining was used to assess the effect of $2 F F$ on cell senescence induced by TNF- $\alpha$, scale bar $=20 \mu \mathrm{m}$. (d) The number of SA- $\beta-G$ al positive cells in chondrocytes treated with or without TNF-a and presence or absence of $2 \mathrm{FF}$ were counted in 5 random fields. (e) The effect of $2 \mathrm{FF}$ on the viability of normal and TNF- $a$ induced chondrocytes $(\mathrm{n}=4)$. The data was represented as Mean $\pm \mathrm{SD}$, $p<0.05$, ** $p$ $<0.01$, and ${ }^{* \star *} p<0.001$. 


\section{Figure 4}

Suppression of FUT10 expression inhibited TNF-a induced expression of OA related proteins, senescence and apoptosis. (a) Relative expression level of 5 of FUTs which involved in synthesis of $a-1,3 / 6$ fucosylation were compared between chondrocytes treated with or without TNF-a for $48 \mathrm{~h}(\mathrm{n}=3)$. (b) Reanalyzed of gene expression data from the published database (GES51588). It demonstrated that the transcription level of FUT10 was significantly up-regulated in medial and lateral tibial of OA patients compared to the healthy controls. (c) Relative expression level of FUT10 from OA patients and normal controls was compared by using qPCR $(n=15)$. (d) Expression of FUT10 in cartilage from OA patients and normal controls were analyzed by western blot assay. $\beta$-tubulin is internal control $(n=4)$. (e) The mRNA level of FUT10 was significantly decreased in chondrocytes transfected with siRNA against FUT10 compared to scramble siRNA $(\mathrm{n}=4)$. (f) After transfected with siRNA-FUT10, the proliferative capability of chondrocytes induced with or without TNF-a were compared by CCK8 assay $(n=4)$. ( $(\mathbf{g})$ After transfected, the senescent cell in chondrocytes treated with or without TNF-a were revealed by SA- $\beta$-Gal staining, scale bar $=20 \mu \mathrm{m}$. (h) The number of SA- $\beta$-Gal positive cells were counted in 5 random fields. The data was represented as Mean $\pm \mathrm{SD}, * p<0.05$, $* \star p<0.01$, and $* \star \star x<0.001$. (i) After transfected, the chondrocytes were stimulated with TNF- $a$, and the apoptosis cells were determined via flow cytometry. (j) Representative immunoblots analysis of MMP-13, IL-1 $\beta$, Caspase- $3 / 8$ and the total and phosphorylated of IKB-a, p65, p38 and JNK in chondrocytes, $\beta$-tubulin is internal control.

\section{Figure 5}

The a-1,3 fucosylation of TNFR1 which mediated by FUT10 impacted the binding capacity to TNF-a. (a) The chondrocytes were transfected with siRNA and treated with or without TNF-a for 48h, and protein level of TNFR1 was determined by Western blot, $\beta$-tubulin is internal control. (b) Immunoblot and lectin blot reactivity of TNFR1 immunoprecipitated from chondrocytes which treated as described above. (c) Immunoblot and lectin blot reactivity of TNFR1 immunoprecipitated from cartilage of OA patients and normal controls. (d) The schematic diagram of the fabrication of TNFR1 antibody microarray. (e) The TNFR1 was immunoprecipitated from chondrocytes transfected with siRNA (low level of a-1,3 fucosylation) or induced with TNF-a (high level of a-1,3fucosylation), and the scanned images are obtained for the analysis of the binding ability of TNFR1 with different level of fucosylatio to TNF- $a$. The spots of TNFR1 antibody were marked with white boxes. (f) The fluorescence intensities of spots were extracted by Genepix 7, and the binding ability of TNFR1 were compared based on foldchange and $t$-test. The data was represented as Mean $\pm \mathrm{SD},{ }^{*} p<0.05$, $* \star p<0.01$, and ${ }^{\star \star *} p<0.001$. 


\section{Supplementary Files}

This is a list of supplementary files associated with this preprint. Click to download.

- Tables2.docx

- TableS3.docx

- Tables4.docx 